\title{
Problem-based Learning Approach to Review the Green Chemistry Principles When Applied to a Polycondensation Reaction
}

\author{
David Salgado-Chavarría*, Joaquín Palacios-Alquisira \\ Laboratorio de Fisicoquímica Macromolecular, Posgrado Facultad de Química, Universidad Nacional Autónoma de México, Ciudad de México, \\ México.
}

*Corresponding Author: davidsalgado.chavarria@gmail.com

\section{ABSTRACT}

A problem-based learning (PBL) methodology was implemented to a project, whose main objectives were to discuss and apply the Twelve Principles of Green Chemistry to the study of poly(vinyl alcohol)'s cross-linking reaction with dicarboxylic acids. The five participating students were oriented to be responsible for their own learning and the professor participated as an advisor. The problem was proposed and students planned all their activities to accomplish the objectives and goals, reviewed recent information in scientific literature and summarized it, made experimental work, prepared written reports, and were evaluated in seminars. The results obtained by the students were assessed through the generation of a final report and also with a final oral presentation in front of faculty members. The experience lived by the collaborative workgroup during the development and execution of the project, is described. This research is an example of how the PBL methodology can motivate the active participation of students when solving problems. The next step is to introduce this tool to teachers and students of other undergraduate courses or laboratories, since it causes a difference in the way education is being perceived in our university, because it emphasizes the application and understanding of concepts over simple memorization.

KEY WORDS: collaborative learning; green chemistry; problem-based learning

\section{INTRODUCTION}

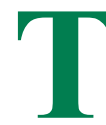
he problem-based learning (PBL) methodology is a student-centered approach that is related to the learning process that occurs when students deal with real world problems, while working in teams to find and develop a solution, with teachers/instructors acting as facilitators (Nagarajan and Overton, 2019). Some elements seem to be common to PBL: Learning is student centered (as mentioned before), problems are structured and authentic, teachers act as advisors, and students work in small groups (Cowden and Santiago, 2016). Although, the elements are in constant interaction, students are responsible of their learning, implying that they have the main role in the cognitive process and should work actively, in group, to solve a problem. On the other hand, instructors act as coaches, they incite group discussion, and they are in charge of monitoring the process. Students are the main characters, since PBL methodology emerged from constructivist learning theories and it was developed as an alternative to conventional teaching (Loyens et al., 2006). Constructivism suggests that humans build knowledge from their experiences and, contrary to traditional education, where students receive knowledge like empty vessels to be filled, in constructivist, students are encouraged to confront what they know (Bada and Olusegun, 2015).

It is evident that, long-term memorability is enhanced by PBL, because it fosters the utilization of previous knowledge to solve a new problem and demands students to put in practice what they have already been taught, therefore, facilitating the comprehension of the concepts (Schmidt et al., 2011). Other benefits that come along with PBL include the improvement of student's creative thinking, self-regulated skills, and selfevaluation (Jansson et al., 2015; Yoon et al., 2014).

Therefore, to improve chemistry student's learning experience, the PBL approach can be used for a better comprehension of the importance of Green Chemistry. According to the U.S. Environmental Protection Agency (EPA, 1990), Green Chemistry is the design of chemical products and processes that reduce or eliminate the use or generation of hazardous substances; this designing process can be assisted by the Twelve Principles of Green Chemistry (Lancaster, 2002). The principles are qualitative, and their aim is to minimize the impact of chemical activities on human health and environment without compromising the chemical process (Ribeiro and Machado, 2013).

There is a commitment to green chemistry education (Armstrong et al., 2018), and efforts have been made to implement it, at the undergraduate level (Timmer et al., 2018; Kennedy, 2016; Manchanayakage, 2013), but there is an uneven development of green chemistry curricular materials, since there have been few comprehensive reforms for general chemistry lecture or laboratory curricula (Armstrong et al., 2019). For example, Green Chemistry has not been covered extensively by chemistry 
textbooks, as most of them have limited coverage of green chemistry ideas (Johnson et al., 2020). For that reason, some authors have considered that a cultural change in chemical education must be done by incorporating green chemistry at the elementary level and continuing throughout graduate coursework (Kitchens et al., 2006) or through new curricula and updated textbooks (Tucker, 2010). Therefore, after considering the significance of Green Chemistry and since high school and university chemistry curricula do not include the study of this important philosophy, we have thought that the utilization of PBL methodology could be applied to improve students' knowledge regarding Green Chemistry.

Therefore, the aim of this work was to present and describe the steps in the implementation of PBL methodology and to summarize the results obtained, by a collaborative group of students, when the Twelve Green Chemistry Principles were applied to the cross-linking polymerization reaction between PVA and different dicarboxylic acids. This work intends to be a working example, so that regardless of the subject, high school and undergraduate teachers could apply the PBL approach in their classrooms.

\section{METHODOLOGY}

The PBL methodology was applied to a group of five Mexican senior students from the Chemistry and Chemical Engineering majors of Universidad Nacional Autónoma de México (UNAM). Students worked at the Laboratory of Macromolecular Physical Chemistry, Facultad de Química (Faculty of Chemistry), which belongs to the same university.

In the first meeting, the five participating students were introduced to the research problem, objectives, and goal; after knowing what it was about, they voluntarily agreed to continue working for 18 weeks scheduled for its completion. On the same meeting, the question was presented: Between conventional heating $(\mathrm{CH})$ and microwave irradiation $(\mathrm{MW})$, which one makes the cross-linking polymerization reaction between poly(vinyl alcohol) and different dicarboxylic acids, greener? Assess the process to obtain the membranes, using the Twelve Principles of Green Chemistry as a guide.

Even though, students were assigned different dicarboxylic acids, this was not an inconvenient for them to work as a team, since the principles of the polymerization technique were basically the same. From this, students gathered and started the planning of the project work, through the preparation of a Gantt chart (activities vs. time).

After completing the Gantt chart, students were very enthusiastic and worked as a team to search in recent literature (i.e., papers, books, and thesis) so that they could know the state of the art of the proposed problem. Two weeks later, and based on the collected information, students were able to identify the variables (dependent and independent) to control the polymerization reaction problem and also they established a reasonable hypothesis; after this, they integrated their protocols, knowing that a good protocol is the starting point of experimental work. At this point, students worked in the laboratory according to what was planned and aiming to find the necessary data to test the hypothesis. It is important to recognize, that during this time, students also advanced on the theoretical framework of their project.

Ten weeks later, participants finished their experimental work, and they were able to accept or reject the hypothesis proposed. After that, they started writing individual final reports, which included all the results regarding the synthesis of the membranes and the discussion about the greenness of the polymerization reactions activated by $\mathrm{CH}$ and $\mathrm{MW}$. Besides results and discussion, the final document also contained objectives, hypothesis, theoretical framework, methodology, and conclusions. The reports were reviewed by the professor and three other faculty members. Students were asked to individually prepare Microsoft PowerPoint presentations, which they used for a final oral examination. The main steps of the methodology used are shown in Figure 1.

There are two remaining points that are important: The first one is that, although in the PBL methodology students are responsible for their own learning and that the professor acts as a facilitator, his role is also meaningful since based on his own experience, he can advise or make suggestions to guide students' work. Our laboratory is convenient in this way, since the professor's desk is in a close area to where students work, then, he was always there for advice. Every 2 weeks the entire group, along with the professor, gathered to discuss their advances and talk about topics in a seminar format. Some of the topics included: the state of the art, the presentation of the protocols, and the presentation of relevant results, among others.

\section{RESULTS AND DISCUSSION}

\section{Learning Outcomes}

The students who participated in the project were able to develop important skills and capabilities, such as teamwork;

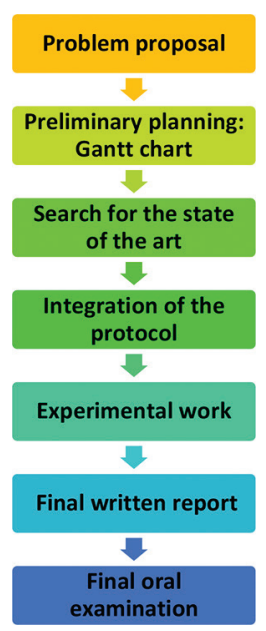

Figure 1: Main steps of the problem-based learning methodology used in this project 
collaboration; analysis, organization, and summarization of the information; critical thinking; problem-solving; and improvement of their oral and written communication skills, along with considering the importance of using green chemistry when designing a process or a product. Figure 2 shows different learning outcomes that were reached by the students.

There are abilities that are gradually developed throughout a person's working life. What is interesting about the PBL methodology, is that it orients students to develop the necessary skills to solve an unknown problem in groups, which is somewhat an introduction of what they will experience in later years, when they enter their working life. Our reflections on some of the learning outcomes are described in the next paragraphs.

\section{Teamwork and Collaboration}

Nowadays, teamwork and collaboration are essential soft skills (Succi and Canovi, 2020), since we simply cannot do everything by ourselves. Here, students helped each other during every step of the process, and it was easier for them to solve the conflicts, since their multiple viewpoints were present during problem solution. Teamwork also fosters the idea of combining skills to finish a task; it was impressive to see that each student has different abilities, which made the team stronger. Therefore, besides the reasons described above, we found teamwork as an exceptional tool for improving learning, sharing the workload, and creating a healthier work environment since stronger relationships were built in the group.

\section{Oral and Written Communication}

Since the team was limited to five students, it was easy for the professor to supervise and help enhancing the quality of the written reports and the oral communication. As part of the PBL methodology, students needed to complete some evaluation products, which included a final report and a final oral examination.

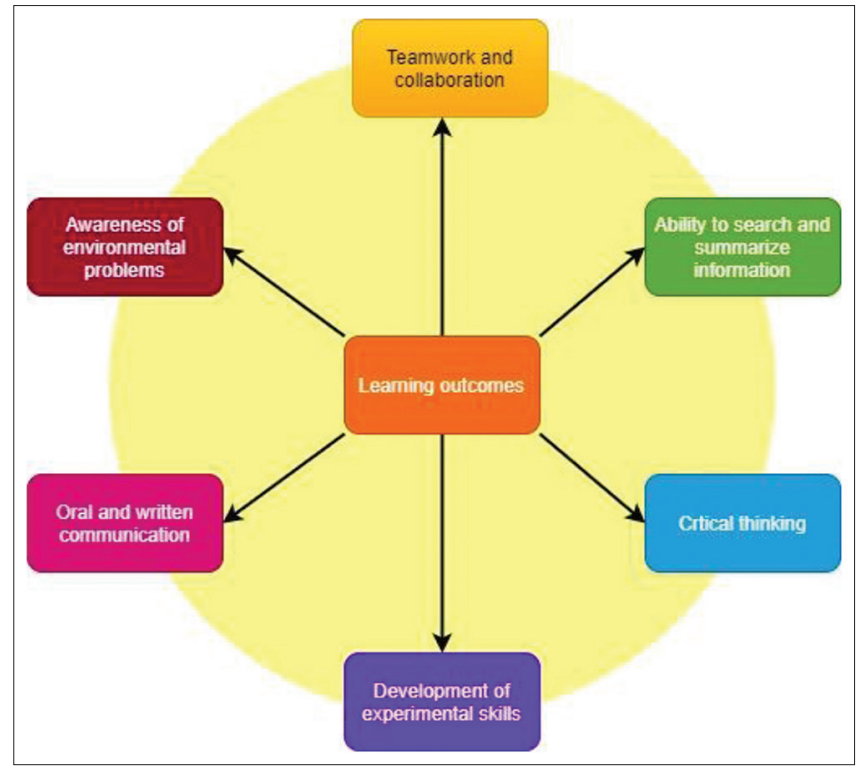

Figure 2: Learning outcomes
The physical evidence of student's work was compiled in a final report. The report was composed by the parts mentioned in the methodology of this paper. The first thing students did, was writing the protocol, then collected the information background of the research, after that, the theoretical framework and finally the results, discussion, and conclusion; meaning that the students worked from the easiest (protocol), to the complete final report. During each step, students handed in the drafts of their written progress to the professor and he corrected possible mistakes. This helped students to improve their writing ability and their critical thinking and analysis, which is especially necessary in the discussion section. The reports were evaluated by other three members of the faculty staff; this also contributed to making a better report.

Regarding oral communication, every 2 weeks, a participant had to present a topic, in a seminar format, which had to be related to the proposed problem. The titles of some of the seminars were:

- Literature review: Poly(vinyl alcohol)'s cross-linking reaction.

- Preparation of membranes of poly(vinyl alcohols) modified with cross-linking agents: Properties and applications.

- Synthesis and characterization of cross-linked poly(vinyl alcohol) membranes.

- Application of the principles of green chemistry to poly(vinyl alcohol)'s cross-linking reaction.

- Assessment of poly(vinyl alcohol)'s cross-linking reaction according to the principles of green chemistry. Comparison between $\mathrm{CH}$ and microwave irradiation as activation media.

Every seminar was $1 \mathrm{~h}$ long. Students prepared the topic and they presented it to the group using Microsoft PowerPoint slides. The professor encouraged participants to ask questions. During the presentation, the professor intervened, orienting discussion, and giving ideas.

As part of their final evaluation, students made an oral presentation in front of three faculty members. The activity consisted in the presentation of results and conclusions obtained by the participants, and then, a round of questions made by each one of the professors.

\section{Awareness of Environmental Problems}

The intention of this project was that students could see the importance of taking into account greener alternatives during the design and synthesis of new materials. The lack of studies on green chemistry by the undergraduate students motivated the professor to create consciousness in the new generation of Mexican chemists and chemical engineers too, which will be in charge of deciding the best conditions and materials to work with, during a production process. The decisions made by them, can have great impact on the environment, therefore compromising the quality of life of the future generations. 


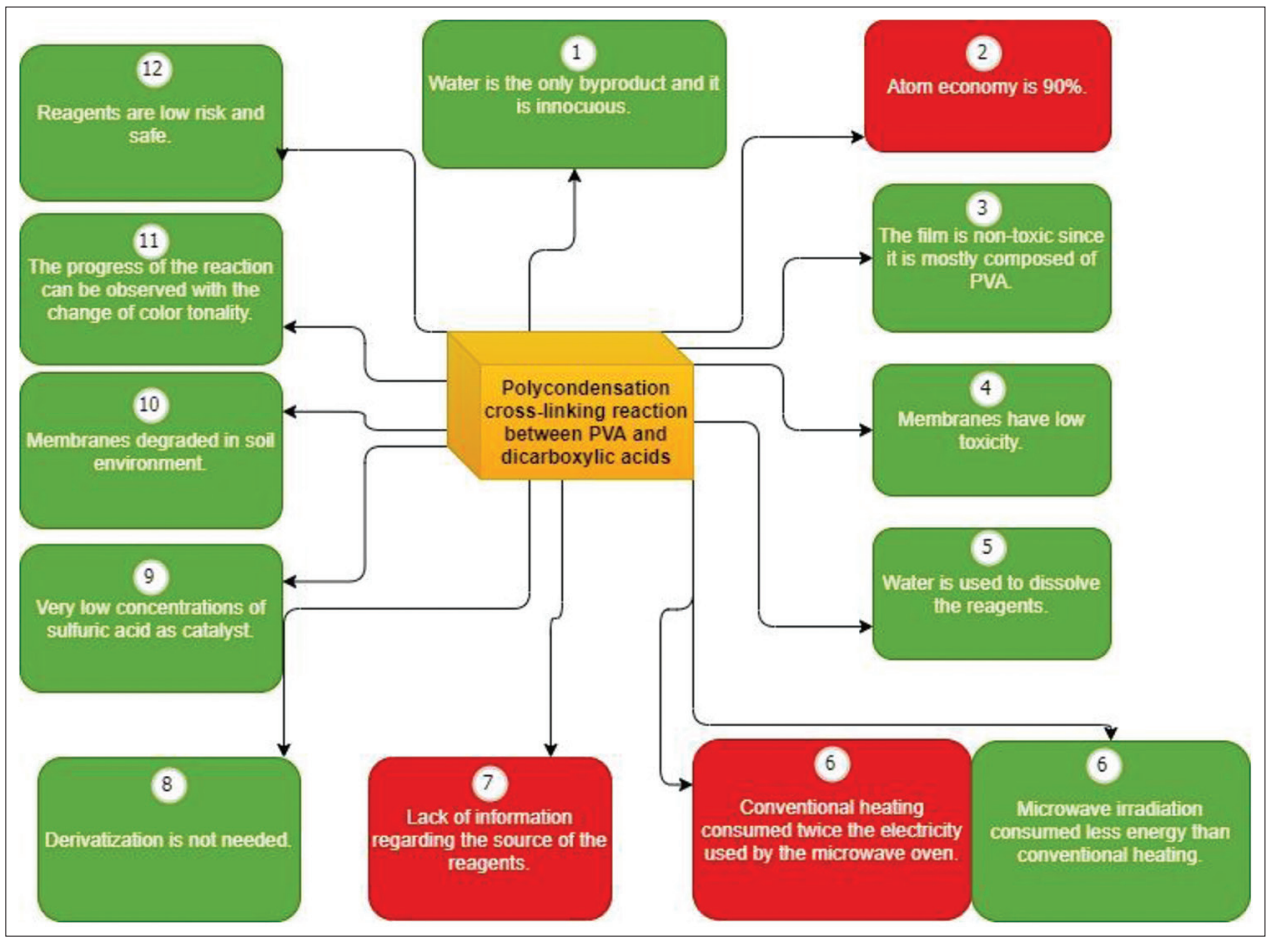

Figure 3: Summary of results and conclusions. Rounded rectangles in green represent the precepts that fulfill the principles of green chemistry; rounded rectangles in red represent the precepts that do not fulfill the principles of green chemistry

After finishing the synthetic process, students discussed, in group, about how green were the two processes: $\mathrm{CH}$ versus MW. Based on scientific information, each student had previously prepared a document with the arguments to decide whether the Green Chemistry Principles were fulfilled or not (Appendix A); Figure 3 contains a summary of results and conclusions of our project.

This type of evaluation could be used by laboratory instructors and teachers from high schools and colleges, since it contributes to students' self-learning, because they have to think about the possible explanations that could prove their thoughts on a certain subject. We also emphasize on the importance of owned experiences since they motivate and open the minds of students to broader and newer experimental possibilities that can be harmless to health or environment.

\section{CONCLUSION}

A PBL methodology was used to increase students' consciousness regarding the importance of green chemistry. The students that participated in the project were able to develop important skills and capabilities, such as teamwork, collaboration, accessing and analyzing information, critical thinking, problem-solving, and improvement of their oral and written communication, along with considering the importance of using green chemistry when designing a process or a product.

Encouraged and guided by their professor, each participant succeeded in the development of the skills mentioned above.
Even though the final evaluation was individual, teamwork was a critical factor for each one of the participants, since the different talents and strengths found in the workgroup were used to promote problem-solving. Seminars were also helpful, since discussion was fostered so that participants could enhance their work and identify failure; at the same time, seminars also intended to prepare students for their final oral examinations.

The discussion made in our workgroup, is an example of how students can apply their knowledge to analyze and compare how green are chemical processes. The PBL methodology, the experimental work and the evaluation of PVA/dicarboxylic acid's system, can be readily used in high school and undergraduate chemistry laboratories since it uses few materials and equipment, and it is also a low-risk experiment, therefore resulting in an excellent exercise for teachers to encourage students' participation in a PBL experience where they can apply and comprehend concepts such as cross-linking reactions, but specially, to learn how to do chemistry based on the observation and analysis of the results.

\section{REFERENCES}

Armstrong, L.B., Rivas, M.C., Douskey, M.C., \& Baranger, A.M. (2018). Teaching students the complexity of green chemistry and assessing growth in attitudes and understanding. Current Opinion in Green and Sustainable Chemistry, 13, 61-67.

Armstrong, L.B., Rivas, M.C., Zhou, Z., Irie, L.M., Kerstiens, G.A., Robak, M.T., Douskey, M.C., \& Baranger, A.M. (2019). Developing a green chemistry focused general chemistry laboratory curriculum: What do students understand and value about green chemistry? Journal of Chemical Education, 96(11), 2410-2419.

Bada, S.O., \& Olusegun, S. (2015). Constructivism learning theory: A 
paradigm for teaching and learning. IOSR Journal of Research and Method in Education, 5(6), 66-70.

Cowden, C.D., \& Santiago, M.F. (2016). Interdisciplinary explorations: Promoting critical thinking via problem-based learning in an advanced biochemistry class. Journal of Chemical Education, 93(3), 464-469.

DeMerlis, C.C., \& Schoneker, D.R. (2003). Review of the oral toxicity of polyvinyl alcohol (PVA). Food and Chemical Toxicology, 41(3), 319-326.

Environmental Protection Agency (EPA). (1990). Pollution Prevention Act of 1990. Washington, DC: Environmental Protection Agency. Available from: https://www.epa.gov/p2/pollution-prevention-act-1990. [Last accessed on 2020 Aug 26].

Jansson, S., Söderström, H., Andersson, P.L., \& Nording, M.L. (2015). Implementation of problem-based learning in environmental chemistry. Journal of Chemical Education, 92(12), 2080-2086.

Johnson, S., Meyers, M., Hyme, S., \& Leontyev, A. (2020). Green chemistry coverage in organic chemistry textbooks. Journal of Chemical Education, 97(2), 383-389.

Kennedy, S.A. (2016). Design of a dynamic undergraduate green chemistry course. Journal of Chemical Education, 93(4), 645-649.

Kitchens, C., Charney, R., Clarens, A., Farrugia, J., Lisowski, C., Naistat, D., Farrugia, J., Clarens, A., O’Neil, A., Lisowski, C., \& Braun, B. (2006). Completing our education. Green chemistry in the curriculum. Journal of Chemical Education, 83(8), 1126.

Lancaster, M. (2002). Green Chemistry: An Introductory Text. Cambridge: The Royal Society of Chemistry.

Loyens, S., Rikers, R., Schmidt, H. (2006). Students' conceptions of constructivist learning: A comparison between a traditional and a problem-based learning curriculum. Advanced in Health Sciences Education, 11, 365-379.

Manchanayakage, R. (2013). Designing and incorporating green chemistry courses at a liberal arts college to increase students' awareness and interdisciplinary collaborative work. Journal of Chemical Education, 90(9), 1167-1171.

Nagarajan, S., \& Overton, T. (2019). Promoting systems thinking using project- and problem-based learning. Journal of Chemical Education, 96(12), 2901-2909.

Ribeiro, M., \& Machado, A. (2013). Holistic metrics for assessment of the greenness of chemical reactions in the context of chemical education. Journal of Chemical Education, 90(4), 432-439.

Salgado-Chavarría, D., \& Palacios-Alquisira, J. (2020). Poly(vinyl alcohol) membranes cross-linked with maleic anhydride and 2,5-furandicarboxylic acid: Conventional heating and microwave irradiation. Chemistry Select, 5(16), 4826-4838.

Schmidt, H.G., Rotgans, J.I., \& Yew, E.H. (2011). The process of problembased learning: What works and why. Medical Education, 45(8), 792-806.

Sonker, A.K., Rathore, K., Nagarale, R.K., \& Verma, V. (2018). Crosslinking of polyvinyl alcohol (PVA) and effect of crosslinker shape (aliphatic and aromatic) thereof. Journal of Polymers and the Environment, 26(5), 1782-1794.

Succi, C., \& Canovi, M. (2020). Soft skills to enhance graduate employability: Comparing students and employers' perceptions. Studies in Higher Education, 45(9), 1834-1847.

Timmer, B.J., Schaufelberger, F., Hammarberg, D., Franzén, J., Ramström, O., \& Dinér, P. (2018). Simple and effective integration of green chemistry and sustainability education into an existing organic chemistry course. Journal of Chemical Education, 95(8), 1301-1306.

Tucker, J.L. (2010). Green chemistry: Cresting a summit toward sustainability. Organic Process Research \& Development, 14(2), 328-331.

Yoon, H., Woo, A.J., Treagust, D., \& Chandrasegaran, A.L. (2014). The efficacy of problem-based learning in an analytical laboratory course for pre-service chemistry teachers. International Journal of Science Education, 36(1), 79-102. 


\section{APPENDIX A}

Green Chemistry Principles review, as applied to PVA's chemical modification reaction, activated by microwave (MW) or conventional heating $(\mathrm{CH})$.

\section{Prevention}

"It is better to prevent waste than to treat or clean up waste after it is formed."

In this case, water is the only by-product of the chemical reaction studied and it is innocuous, it does not represent a hazard for health and/or environment, therefore, we considered that this precept is accomplished as an important part of the concepts of green chemistry.

\section{Atom Economy}

"Synthetic methods should be designed to maximize the incorporation of all materials used in the process into the final product."

Atom economy is essentially a measure of how many atoms of reactants end up in the final product and how many end up in by-products or waste.

For PVA's cross-linking reaction, atom economy is $90 \%$, which is good if we consider that the by-product is water, nevertheless, this principle demands $100 \%$ compliance of the atom economy, then, the reaction that involves the modification of the polymer does not fully accomplish this principle of green chemistry.

\section{Less Hazardous Chemical Synthesis}

"Whenever practicable, synthetic methodologies should be designed to use and generate substances that pose little or no toxicity to human health and the environment."

The dicarboxylic acid is incorporated to PVA through a crosslinking reaction, the released by-product is water. After reaction is complete, the main product is a cross-linked polymer, which is composed by ester groups and also by unreacted hydroxyl groups from PVA, then, this film can be considered as nontoxic or with a very low toxicity, since it is mostly composed of PVA (DeMerlis and Schoneker, 2003). Thus, this precept complies with the green chemistry principles.

\section{Designing Safer Chemicals}

"Chemical products should be designed to preserve efficacy of the function while reducing toxicity."

The membranes have low toxicity since most of the product is PVA, as explained above. Then, this precept complies with green chemistry.

\section{Safer Solvents and Auxiliaries}

"The use of auxiliary substances (e.g., solvents or separation agents, etc.) should be made unnecessary whenever possible and, when used, innocuous."
As seen in a previous work (Salgado-Chavarría and PalaciosAlquisira, 2020), water was used as a solvent for this reaction, but before starting the cross-linking process, the solvent evaporated at room temperature for $24 \mathrm{~h}$, then, the reaction is performed as a solid-state polymerization, which, in other words, is solvent free. These types of reactions are considered as the ideal situation, since the risk of hazard is reduced, and solvents are avoided. This principle is accomplished.

\section{Design for Energy Efficiency}

"Energy requirements of chemical processes should be recognized for their environmental and economic impacts and should be minimized. If possible, synthetic methods should be conducted at ambient temperature and pressure."

The study showed that the process using MW activation, spent only one-eighth of the total time used by the convective heating; along with this, the oven used to conventionally cross-link the samples, consumed $8.52 \times 10^{-2} \mathrm{kWh}$ versus $4.01 \times 10^{-2} \mathrm{kWh}$ that were used by the MW oven. The reduction in energy consumption is 2 times lower for MW activation, then, the PVA cross-linking reaction, activated by $\mathrm{MW}$, is greener than $\mathrm{CH}$-assisted process.

\section{Use of Renewable Feedstocks}

"A raw material or feedstock should be renewable rather than depleting whenever technically and economically practicable."

Even though there are greener alternatives to produce PVA as well as the different dicarboxylic acids, this precept was considered to not comply with the principles of green chemistry, due to the lack of information regarding the source of the reagents used in the cross-linking reaction.

\section{Reduce Derivatives}

"Unnecessary derivatization (use of blocking groups, protection/deprotection, and temporary modification of physical/chemical processes) should be minimized or avoided if possible, because such steps require additional reagents and can generate waste."

In the studied synthetic process, there is no need for derivatization, since the cross-linking reaction between PVA's hydroxyl groups and the dicarboxylic acids proceeds directly to esterification reaction, then, the principle is accomplished.

\section{Catalysis}

"Catalytic reagents (as selective as possible) are superior to stoichiometric reagents."

Sulfuric acid was used as a catalyst in the cross-linking reaction between PVA and the dicarboxylic acids. The amount of acid used during experimental work was very low and it does not represent an important issue for the sustainability of the process, therefore, this principle meets the green chemistry criteria. 


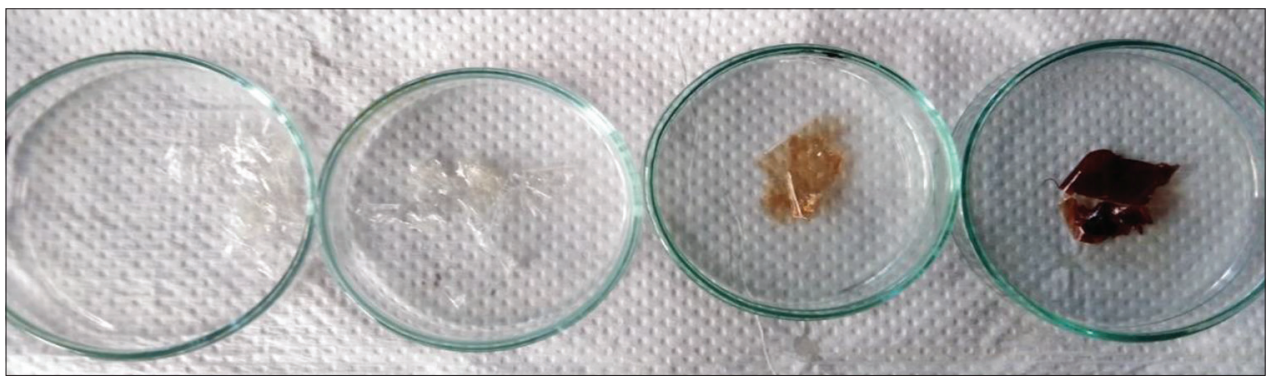

Figure a1: Poly(vinyl alcohol)/maleic-anhydride membranes. Change in color due to longer cross-linking reaction times, maleic anhydride initial concentration remained constant. From left to right: $0.5 \mathrm{~h}, 0.75 \mathrm{~h}, 1 \mathrm{~h}$, and $2 \mathrm{~h}$ of cross-linking reaction time and using conventional heating activation

\section{Design for Degradation}

"Chemical products should be designed so that at the end of their function they break down into innocuous degradation products and do not persist in the environment."

Although the current experimental work did not conduct any biodegradability test, it has been found in similar studies (Sonker et al., 2018), that after 60 days of biodegradation experiments, PVA cross-linked membranes degrade in soil environment. Similar results are expected to occur with our membranes; therefore, the principle is accomplished.

\section{Real-time Analysis for Pollution Prevention}

"Analytical methodologies need to be further developed to allow for real-time, in-process monitoring and control prior to the formation of hazardous substances."

As the cross-linking reaction proceeds, a special analytical assessment was not necessary since the progress of the reaction is observed with the change of membrane color tonality. The films change from clear transparent to dark amber color, meaning that the esterification reaction can be observed by a colorimetric evaluation. This is an interesting and helpful tool for practical laboratory work since students can follow how fast the new material is formed just by simple color change observation. As shown in Figure a1, at lower cross-linking reaction times, samples do not show a change in tonality, but as the sample is held more time in the oven, it becomes darker, as an indication that cross-linking reaction occurred, therefore, this principle is accomplished.

\section{Inherently Safer Chemistry for Accident Prevention}

"Substances in the form of a substance used in a chemical process should be chosen to minimize the potential for chemical accidents, including releases, explosions, and fires."

In every experimental work done in the laboratory, personal protective equipment (PPE) must be worn. However, in this experiment, the risk is reduced due to the low toxicity, flammability, and amount of the reagents used, therefore, this precept complies with the principles of green chemistry. 